\title{
Cáncer de colon sigmoides como contenido de una hernia inguinal izquierda
}

Daniel Alfredo González González*, Nicolás Tarigo†

\section{Resumen}

El cáncer de colon como contenido de una hernia inguinal es una situación infrecuente. Pocos casos se han reportado en la literatura. Habitualmente ocurre en hernias inguinales izquierdas y es el colon sigmoides su contenido. La palpación de una tumoración en una hernia que previamente no existía y la aparición de sintomatología intestinal orientan el diagnóstico. El colon por enema constituye el examen paraclínico por excelencia para su confirmación. El tratamiento quirúrgico se impone, sin embargo son posibles diversas tácticas y vías de abordaje.

Se presenta el caso clínico de un paciente de 82 años con un cáncer de sigmoides como contenido de una hernia inguinal que se presentó con una tumoración sobre la hernia y alteraciones del tránsito intestinal. La ecografía de la región orientó el diagnóstico y el colon por enema confirmó una lesión intrínseca colónica. Se resolvieron ambas enfermedades por un abordaje inguinal, realizándose la sigmoidectomía oncológica y la herniorrafia sin complicaciones con una resección oncológicamente satisfactoria.

Palabras clave: HERNIA INGUINAL NEOPLASIAS DEL COLON

Key words: INGUINAL HERNIA COLONIC NEOPLASMS

\section{Introducción}

La presencia de un cáncer de colon como contenido de una hernia inguinal es una situación infrecuente, habiéndose publicado menos de 50 casos en la literatura hasta el momento actual ${ }^{(1-8)}$, habiendo sido uno de ellos comunicado en Uruguay por Palacio ${ }^{(9)}$ en el año 1993.

El objetivo de este trabajo es comunicar un nuevo caso de cáncer de colon sigmoides como contenido de una hernia inguino escrotal izquierda.

\section{Caso clínico}

Paciente de 82 años, sexo masculino, portador de hernia inguinal bilateral de años de evolución, sin historia de sufrimiento, que consultó por la aparición de una tumefacción sobre la hernia inguinal izquierda. Concomitantemente refirió una historia dada por alternancia constipación diarrea.

Al examen buen estado general, buen estado nutricional, bien coloreado.

Hernia inguino escrotal bilateral, irreductible. A nivel de la hernia izquierda se palpó una tumoración pétrea, de 2 por $2 \mathrm{~cm}$ aproximadamente, móvil, no dolorosa. Testículo y cordón de características normales. Examen proctológico: no se palparon tumoraciones. Se realizó una ecografía de partes blandas que informó lesión compatible con tumor de colon. Con planteo de cáncer de colon se solicitó un colon por enema que evidenció una lesión estenosamente del sigmoides en la hernia (figuras 1 y 2). Resto del estudio normal.

No se constató diseminación a distancia mediante TAC tóraco abdómino pélvica.

CEA: $1,89 \mathrm{ng} / \mathrm{ml}$.

\footnotetext{
* Profesor Agregado de Clínica Quirúrgica. Clínica Quirúrgica 3. Hospital Maciel. Montevideo, Uruguay. † Asistente de Clínica Quirúrgica. Clínica Quirúrgica F. Hospital de Clínicas. Montevideo, Uruguay Correspondencia: Daniel Alfredo González González: Atanasio Sierra 3653, Florida. Uruguay. CP 94000. Correo electrónico: danielalfredogg@gmail.com

Recibido: 8/3/17

Aprobado: 9/6/17
} 


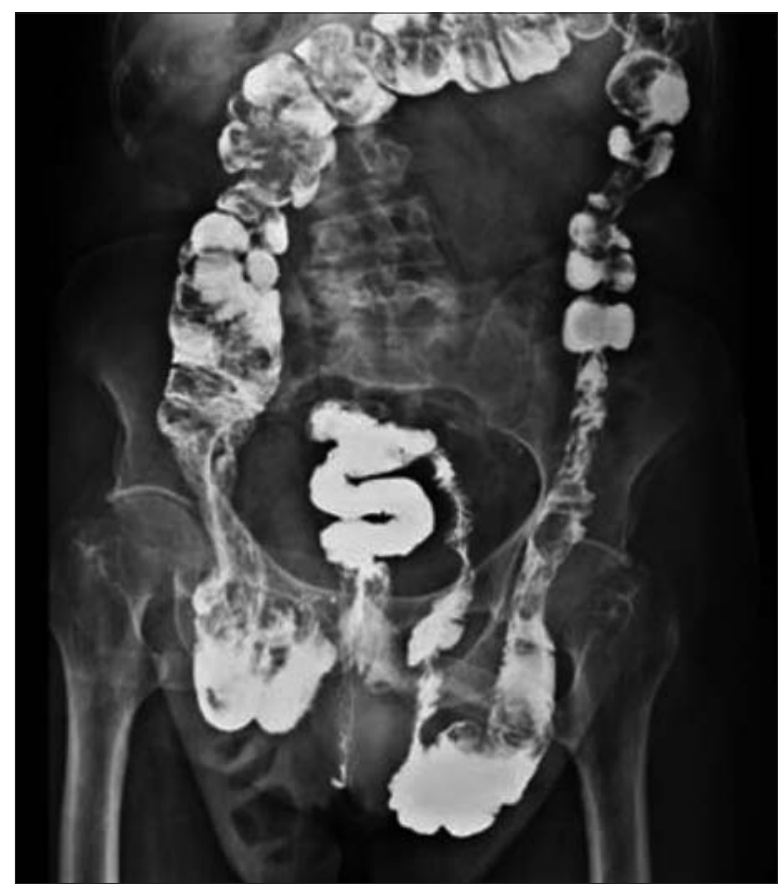

Figura 1. Colon por enema. Hernia inguinal bilateral con contenido colónico.

Se operó de coordinación. Se realizó un abordaje inguinal, se constató la presencia de la tumoración sobre colon sigmoides largo y libre. Se realizó la sigmoidectomía oncológica y anastomosis. Finalmente se repararon ambas hernias mediante herniorrafias.

Buena evolución posoperartoria.

La anatomía patológica confirmó que se trataba de un adenocarcinoma bien diferenciado y la estadificación posoperatoria determinó un estadio I.

A 26 meses de la cirugía la enfermedad oncológica se encuentra controlada y las reparaciones parietales continentes.

\section{Discusión y comentarios}

De acuerdo a la revisión de la literatura, los cánceres de colon contenidos en una hernia inguinal se presentan mayoritariamente en hombres entre la octava y novena década de la vida. En general se destaca el antecedente personal de una hernia inguinal de años de evolución que en determinado momento se hace irreductible, agregando una tumoración sobre la región herniaria que previamente no existía y en muchas oportunidades sintomatología del tracto digestivo bajo. Otros lo hacen con una complicación, habitualmente estrangulación o perforación dentro del saco herniario ${ }^{(5,10)}$.

Los cánceres de sigmoides son los más frecuentemente encontrados como contenido de una hernia ingui-

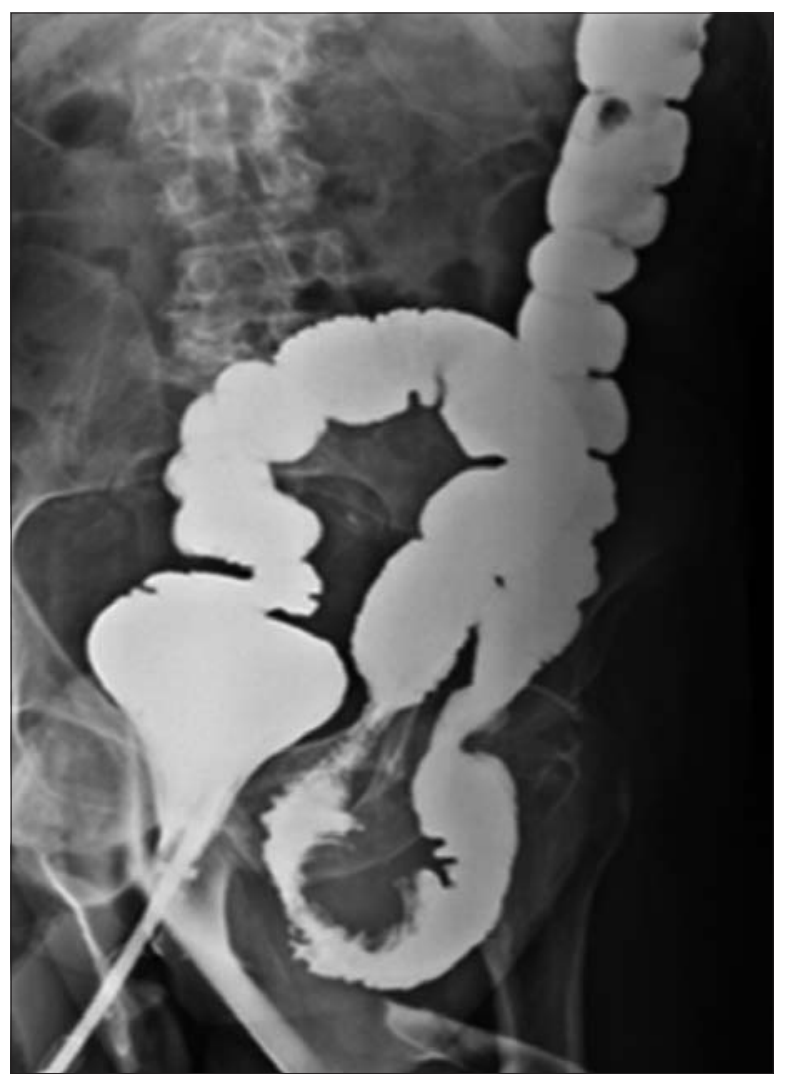

Figura 2. Colon por enema. Imagen de sustracción en colon sigmoides contenido en la hernia izquierda.

nal izquierda. Falides ${ }^{(1)}$ lo atribuye a la longitud y movilidad de esta víscera en relación con el ascendente.

El examen clínico constituye un elemento fundamental de sospecha cuando se palpa una tumoración sobre una hernia inguinal que previamente no existía y clínicamente el contenido es intestinal, siendo las estructuras del cordón y testículo de características normales. De acuerdo a la clasificación de Lejar ${ }^{(11)}$, los tumores en una hernia pueden clasificarse en: 1) Saculares, cuando los mismos se originan en las estructuras propias del saco (tumores primarios de peritoneo o carcinomatosis peritoneal), de los cuales se han comunicado secundarios a cánceres gástricos, colónicos y adenocarcinomas primarios de peritoneo ${ }^{(12-15)}$. 2) Intrasaculares determinados por tumores primarios de los órganos contenidos en el saco, tal el caso presentado.

Desde el punto de vista imagenológico, la ecografía constituye una herramienta importante si es realizada por un especialista con experiencia, ya que descarta enfermedades del cordón espermático y testículo, y puede sugerir su origen colónico. Sin dudas, el colon por enema en esta situación constituye el estudio diagnóstico por excelencia pues permite visualizar el colon contenido en el saco y además las características de la lesión 
que, en general, se trata de zonas de extensión variable de estenosis irregulares de la luz colónica. La fibrocolonoscopía tiene el inconveniente de no poder explorar el colon intrasacular por la imposibilidad de progresar en el bucle determinado por el intestino dentro de la hernia.

Con respecto al tratamiento, la discusión se centra en la vía de abordaje y la táctica a adoptar en la reparación parietal inguinal. El abordaje puede ser inguinal o abdominal. Con respecto a la vía inguinal la ventaja es que se puede resolver ambas enfermedades por una única vía y evitar una laparotomía. La condición es realizar un tratamiento oncológicamente satisfactorio, para lo cual es necesario contar con un asa sigmoidea amplia ${ }^{(3,6)}$. Como desventaja se encuentra la eventual infección del sitio quirúrgico, ya que se trata de una cirugía limpia contaminada (cirugía colónica) y otra limpia (cirugía de la hernia inguinal) y en esta situación debe evitarse la realización de una hernioplastia y optar por una herniorrafia sabiendo su mayor porcentaje de recidiva. En nuestro caso optamos por ésta, ya que se trató de un paciente de 82 años que no realiza actividades de esfuerzo, por lo que la probabilidad de recidiva se reduce. En referencia a la infección del sitio quirúrgico cuando se trata de una herniorrafia es posible su manejo con curaciones y antibióticos, en cambio, de ocurrir en una herniolastia, podría obligar al retiro de la malla.

También se ha propuesto el abordaje laparotómico a través de una incisión mediana y resolución de la patología parietal por vía intraperitoneal ${ }^{(1)} \mathrm{o}$ abordaje inguinal. Otros postulan una incisión transversa de fosa ilíaca izquierda ${ }^{(16)}$.

También se ha reportado la resolución por abordaje laparoscópico del cáncer de colon y reparación por vía convencional de la hernia ${ }^{(17,18)}$. Si bien no hay ningún caso reportado, la resolución por vía laparoscópica de ambas enfermedades es posible.

\section{Abstract}

Carcinoma of the colon in an incarcerated inguinal hernia is rather an unusual condition. Few cases have been reported in literature. Usually it appears in left inguinal hernias and involves the sigmoid colon. Palpation of a tumor in a hernia that did not exist previously and the presentation of intestinal symptoms aid diagnosis. Barium enema constitutes the gold standard in paraclinical studies to confirm diagnosis. Surgical treatment is advised, although several tactics and approaches are possible.

The study presents the clinical case of an 82 year-old patient with sigmoid colon cancer in an incarcerated left inguinal hernia which appeared as a tumor in the hernia and alterations in the intestinal alterations. The ultrasound suggested a possible diagnosis and the Barium enema confirmed the intrinsic colon lesion. Both lesions were solved by an inguinal approach. An oncological sigmoidectomy was performed and herniorrhaphy with no complications with a satisfactory oncological resection was achieved.

\section{Resumo}

O carcinoma de cólon em hérnia inguinal encarcerada é um achado pouco comum. Poucos casos estão relatados na literatura. De maneira geral aparece nas hérnias inguinais esquerdas e inclui o cólon sigmoide. in an incarcerated inguinal hernia is rather an unusual condition. Few cases have been reported. A palpação de um tumor em uma hérnia que não existia anteriormente e a presença de sintomas intestinais ajudam o diagnóstico. O enema de bário é o gold standard dos exames auxiliares para confirmar o diagnóstico. Recomenda-se o tratamento cirúrgico, porém existem várias formas de abordagem e táticas.

Este estudo descreve o caso clínico de um paciente com 82 anos com um câncer de cólon sigmoide em hérnia inguinal que se apresentou como tumor na hérnia e alterações intestinais. O ultrassom sugeria um possível diagnóstico e o enema de bário confirmou a lesão do cólon. Ambas lesões foram solucionadas em uma abordagem inguinal. Realizou-se sigmoidectomia oncológica e herniarrafia sem complicações com ressecção oncológica.

\section{Bibliografía}

1. Falidas E, Gourgiotis S, Papachrysostomou M, Oikonomou C, Villias C. Sigmoid carcinoma localized in the sac of a right inguinoscrotal hernia. J Nat Sci Biol Med 2015; 6(Suppl 1):S150-2.

2. Meniconi RL, Vennarecci G, Lepiane P, Laurenzi A, Santoro R, Colasanti M, et al. Locally advanced carcinoma of the cecum presenting as a right inguinal hernia: a case report and review of the literature. J Med Case Rep 2013; 7:206.

3. Ruiz-Tovar J, Ripalda E, Beni R, Nistal J, Monroy C, Carda P. Carcinoma of the sigmoid colon in an incarcerated inguinal hernia. Can J Surg 2009; 52(2):E31-E32.

4. Mai CM, Chen CY, Hsu KF, Hsiao CW, Jao SW, Wu CC. Colonic neoplasm in a strangulated inguinal hernia. Rev Esp Enferm Dig 2010; 102(5):339-41.

5. Ko KH, Yu CY, Kao CC, Tsai SH, Huang GS, Chang WC. Perforated sigmoid colon cancer within an irreducible inguinal hernia: a case report. Korean J Radiol 2010; 11(2):231-233.

6. Slater R, Amatya U, Shorthouse AJ. Colonic carcinoma presenting as strangulated inguinal hernia: report of two cases and review of the literature. Tech Coloproctol 2008; 12(3):255-8. 
7. Nárožný V. [Carcinoma of the sigmoid colon in an incarcerated inguinal hernia]. Rozhl Chir 2014; 93(11):554-6. [Article in Czech].

8. Gross BH, Botsford JA, Colley DP. Colon carcinoma in a right inguinal hernia. J Can Assoc Radiol 1980; 31(2):145-6.

9. Palacio JL, Cluzet O, Font F, Müller A, El-Ters A. Cáncer de colon como contenido de una hernia inguinal. Cir Urug 1993; 63(1-2-3):80-2.

10. Tan A, Taylor G, Ahmed T. Perforated sigmoid colon carcinoma in an irreducible inguinoscrotal hernia. Ann R Coll Surg Engl 2013; 95(7):125-6.

11. Lejar J. Neoplasmes herniaires et peri-herniaires. Gaz Hosp $1889 ; 62: 801-11$.

12. Oruc MT, Kulah B, Saylam B, Moran M, Albayrak L, Co'kun F. An unusual presentation of metastatic gastric cancer found during inguinal hernia repair: case report and review of the literature. Hernia 2002; 6(2):88-90.
13. Matsumoto G, Ise H, Inoue H, Ogawa H, Suzuki N, Matsuno $\mathrm{S}$. Metastatic colon carcinoma found within an inguinal hernia sac: report of a case. Surg Today 2000; 30(1):74-7.

14. Assarian A, Zaidi AZ, Raja MA. Primary peritoneal adenocarcinoma detected within a femoral hernia sac. Hernia 2005; 9(2):192-4.

15. Roslyn JJ, Stabile BE, Rangenath C. Cancer in inguinal and femoral hernias. Am Surg 1980; 46(6):358-62.

16. Tan GY, Guy RJ, Eu KW. Obstructing sigmoid cancer with local invasion in an incarcerated inguinal hernia. ANZ J Surg 2003; 73(1-2):80-2.

17. Kanemura T, Takeno A, Tamura S, Okishiro M, Nakahira S, Suzuki R, et al. Elective laparoscopic surgery for sigmoid colon carcinoma incarcerated within an inguinal hernia: report of a case. Surg Today 2014; 44(7):1375-9.

18. Carr WR, O'Dair G. A laparoscopic high anterior resection for sigmoid cancer with extraction through incarcerated left inguinal hernia repaired with Permacol mesh. BMJ Case Rep 2012: published online 21 September 2012. 